\title{
Indications of anisotropy at large angular scales in the arrival directions of cosmic rays detected at the Pierre Auger Observatory
}

\author{
Imen Al Samarai ${ }^{* a}$ for the Pierre Auger Collaboration ${ }^{b}$ \\ ${ }^{a}$ LPNHE, CNRS/IN2P3 \& Université Pierre et Marie Curie, Paris, France \\ ${ }^{b}$ Observatorio Pierre Auger, Av. San Martín Norte 304, 5613 Malargüe, Argentina \\ E-mail: auger_spokespersons@fnal.gov \\ Full author list: http://www.auger.org/archive/authors_2015_06.html
}

\begin{abstract}
The large-scale distribution of arrival directions of high-energy cosmic rays carries major clues to understanding their origin. The Pierre Auger Collaboration have implemented different analyses to search for dipolar and quadrupolar anisotropies in different energy ranges spanning four orders of magnitude. A common phase $\approx 270^{\circ}$ of the first harmonic modulation in right-ascension was found in adjacent energy intervals below $1 \mathrm{EeV}$, and another common phase $\approx 100^{\circ}$ above 4 $\mathrm{EeV}$. A constancy of phase measurements in ordered energy intervals originating from a genuine anisotropy is expected to appear with a smaller number of events than those needed to achieve significant amplitudes. This led us to design a prescribed test aimed at establishing whether this consistency in phases is real at $99 \% \mathrm{CL}$. The test required a total independent exposure of $21,000 \mathrm{~km}^{2} \mathrm{sryr}$. We report on the status of this prescription. We also report the results of the search for a dipole anisotropy for cosmic rays with energies above $4 \mathrm{EeV}$ using events with zenith angles between $60^{\circ}$ and $80^{\circ}$. Compared to previous analyses of events with zenith angles smaller than $60^{\circ}$, this extension increases the size of the data set by $30 \%$, and enlarges the fraction of exposed sky from $71 \%$ to $85 \%$. The largest departure from isotropy is found in the energy range above $8 \mathrm{EeV}$, with an amplitude for the first harmonic in right ascension $r_{1}=(4.4 \pm 1.0) \times 10^{-2}$, that has a chance probability $P\left(\geq r_{1}\right)=6.4 \times 10^{-5}$, reinforcing the hint previously reported with vertical events alone.
\end{abstract}

The 34th International Cosmic Ray Conference,

30 July- 6 August, 2015

The Hague, The Netherlands

\footnotetext{
* Speaker.
} 


\section{Introduction}

Establishing the energy at which the flux of extragalactic Cosmic Rays (CRs) starts to dominate the flux of Galactic ones would provide an important step forward in understanding the demands that must be placed upon their accelerators in the Galaxy, and the origin of ultra-high energy CRs. To this aim, the large-scale distribution of arrival directions of CRs as a function of their energy is a relevant tool to study generic signatures of anisotropies in the framework of Galactic scenarios around $1 \mathrm{EeV}$, and extragalactic ones at the highest energies.

The Pierre Auger Observatory [1] provides the largest number of events ever collected to scrutinise such anisotropies in this energy range. The Observatory combines two techniques to detect the extensive air showers resulting from the interaction of CRs with the atmosphere. The longitudinal development of the air showers is measured by fluorescence detectors, while the lateral distribution of the secondary particles at ground level is measured by the Surface Detector Array (SD). Two arrays of SDs are operating: an arrangement of water Cherenkov detectors separated by $1500 \mathrm{~m}$ distributed over an area of $3000 \mathrm{~km}^{2}$, reaching full detection efficiency at $3 \times 10^{18} \mathrm{eV}$, and an array with detectors separated by $750 \mathrm{~m}$ distributed over an area of $23.5 \mathrm{~km}^{2}$, reaching full detection efficiency at $3 \times 10^{17} \mathrm{eV}$.

The different analysis methods used in determining the amplitude and phase of the modulation in Right Ascension (RA) in different energy bins are described in section 2. Using data from both the $750 \mathrm{~m}$ array and the $1500 \mathrm{~m}$ array up to the end of 2014, we present in section 3 the updated status of a prescribed test on the phases of the first harmonic modulation in RA previously reported in [2]. Studies of the flux modulation in declination and in RA from the analysis of events with zenith angles smaller than $60^{\circ}$ have been reported in $[3,4]$. By including events with zenith angles between $60^{\circ}$ and $80^{\circ}$, an increase of $30 \%$ in the number of events is obtained [5]. In section 4, we present the results of the reconstructed dipolar modulations in RA and in declination above $4 \mathrm{EeV}$ for this data set. Finally, the complete picture of large-scale anisotropy studies over four decades in energy using the Pierre Auger Observatory data is presented in the last section.

\section{Harmonic analysis in right ascension}

Harmonic analysis of the RA distribution is a classical tool for picking up and for characterising any modulation in this coordinate system [6]. The low amplitudes of the first harmonic modulation expected from the limits set in many historic studies, motivate a careful consideration of possible spurious modulations that arise from experimental or atmospheric effects. In particular, due to the steepness of the energy spectrum, even small changes in the energy estimator as a function of time or the local angular coordinates would distort significantly the counting rate of events above a given energy.

Atmospheric conditions are known to affect the observed shower size for a given primary energy. For a larger (smaller) pressure, an air shower will be at a more (less) advanced stage of development when it arrives at the ground since the column density traversed would be larger (smaller). Also the air density affects the Molière radius and hence the lateral profile of the showers. These atmospheric effects are accounted for by correcting the energy estimator of the events according to 
the weather conditions at the time each event was recorded [7]. Such corrections guarantee that the observed event rate with time is controlled by the instantaneous exposure only.

Rayleigh analysis. For a directional exposure $\omega(\alpha)$, where $\alpha$ is the RA, the flux $\Phi(\alpha)$ can be decomposed in terms of a harmonic expansion from the observed distribution of arrival directions $d N / d \alpha$ as:

$$
\Phi(\alpha)=\frac{1}{\omega(\alpha)} \frac{d N}{d \alpha}=a_{0}^{\alpha}+\sum_{n>0} a_{n}^{\alpha} \cos n \alpha+\sum_{n>0} b_{n}^{\alpha} \sin n \alpha .
$$

The first harmonic coefficients $(n=1)$ in RA are enough to reconstruct the equatorial dipole component under the hypothesis that contributions from higher-order multipoles are negligible, while the second harmonic coefficients $(n=2)$ are sensitive to the quadrupole component (and to eventual higher-order multipoles). The Fourier coefficients of the flux can be estimated as:

$$
a_{n}^{\alpha}=\frac{2}{\tilde{N}} \sum_{i=1}^{N} w_{i} \cos \left(n \alpha_{i}\right), b_{n}^{\alpha}=\frac{2}{\tilde{N}} \sum_{i=1}^{N} w_{i} \sin \left(n \alpha_{i}\right),
$$

where the sums run over the number of events $N$ in the energy range considered, and the normalization factor is $\tilde{N}=\sum_{i=1}^{N} w_{i}$. The factors $w_{i}$ account for the variations in the operating size of the array as a function of time that lead to small modulations in the exposure. Their determination is optimised within the energy range where the study is performed and is detailed in the following sections. The amplitude can then be expressed as $r_{n}=\sqrt{\left(a_{n}^{\alpha}\right)^{2}+\left(b_{n}^{\alpha}\right)^{2}}$, and the phase as $\phi_{n}=1 / n \arctan \left(b_{n}^{\alpha} / a_{n}^{\alpha}\right)$. In case of an underlying isotropy, the amplitude follows a Rayleigh distribution while the phase follows a uniform distribution. The probability $\mathrm{P}\left(\geq r_{n}\right)$ that an amplitude equal to or larger than $r_{n}$ arises from an isotropic distribution can be approximated by the cumulative distribution function of the Rayleigh distribution $P\left(\geq r_{n}\right)=\exp \left(-\tilde{N} r_{n}^{2} / 4\right)$.

East-West analysis. Alternatively to modelling the event rate and controlling the variations of the exposure with time, the modulation of the flux in RA can be revealed using the East-West (E-W) method, though it is less sensitive than the Rayleigh analysis by a factor $\simeq 2.5$ [8]. The counting rate of the events observed in either the Eastern or the Western half field of view of the array is subjected to variations during a sidereal day that can be either due to experimental effects and/or to real variations in the primary CR flux from different parts of the sky. Systematic effects of experimental origin are independent of the incoming direction so that they can be removed by subtracting the counting rates of events coming from each of the sectors. On the other hand, in the presence of a genuine equatorial dipole, the difference in the E-W counting rate would show modulations that are expected to be related to those of the genuine dipole. More specifically, the amplitude $r_{E W}$, and phase $\phi_{E W}$ can be calculated from the arrival times of $N$ events using the standard first harmonic analysis, slightly modified to account for the subtraction of the Western sector to the Eastern one [8]. The amplitude of the first harmonic, $r_{1}$, and the phase, $\phi_{1}$, of the RA modulation determined with the Rayleigh formalism are related to $r_{E W}$ and $\phi_{E W}$ through the relations $r_{1}=r_{E W} \pi\langle\cos \delta\rangle / 2\langle\sin \theta\rangle$, and $\phi_{1}=\phi_{E W}+\pi / 2$ [8], where $\langle\cos \delta\rangle$ is the mean value of the cosine of the declinations of events, and $\theta$ is the zenith angle.

Reconstruction of the equatorial dipole component. In the case of an underlying pure dipole, the relationship between $r_{1}$ and the projection of the dipole on the Earth equatorial plane $d_{\perp}$, which is the quantity of interest to compare the results of different experiments, depends on the latitude of the observatory and on the range of zenith angles considered. To first order, the 
relationship reads $d_{\perp} \simeq r_{1} /\langle\cos \delta\rangle$, with $\langle\cos \delta\rangle \simeq 0.78$ for events with zenith angles below $60^{\circ}$ at the Auger Observatory [9].

\section{Phase of the first harmonic from $\simeq 10 \mathrm{PeV}$ to the highest energies}

Harmonic analyses in RA have been previously reported in [2] and [9]. Although no significant departure from isotropy was revealed in the amplitude, notable features concerning the evolution of the phases with energy from $10^{16} \mathrm{eV}$ up to the highest energies were pointed out. In this section, we report on the status of these features with independent data.

In [9], a Rayleigh analysis of the data collected at the $1500 \mathrm{~m}$ array above $1 \mathrm{EeV}$ was presented in detail. In this analysis, the weight factors $w_{i}$ make use of the number of active cells $\mathrm{n}_{\text {cell }}(t)$ (number of active detectors surrounded by six active neighbours) constantly monitored at the Observatory. The total number of active cells, $N_{\text {cell }}$, as a function of the sidereal time $\alpha_{0}$ (measured by the right ascension of the zenith at the center of the array) and its relative variations, $\Delta N_{\text {cell }}$, are obtained as $N_{\text {cell }}\left(\alpha_{0}\right)=\sum_{j} n_{\text {cell }}\left(\alpha_{0}+j . T_{\text {sid }}\right)$ and $\Delta N_{\text {cell }}\left(\alpha_{0}\right)=\frac{N_{\text {cell }}\left(\alpha_{0}\right)}{\left\langle N_{\text {cell }}\right\rangle}$, where $T_{\text {sid }}$ is the duration of the sidereal day, and $\left\langle N_{\text {cell }}\right\rangle=T_{\text {sid }}^{-1} \int_{0}^{T_{\text {sid }}} d \alpha_{0} N_{\text {cell }}\left(\alpha_{0}\right)$. The weighting factor for each event is thus derived as $w_{i}=\Delta N_{\text {cell }}^{-1}\left(\alpha_{0}^{i}\right)$. Below $1 \mathrm{EeV}$, additional spurious modulations of the event rate arise from the variation of the detection efficiency with time (through the impact of weather effects). For this reason, amplitudes and phases are derived from the E-W method. In [2], amplitudes and phases as obtained from the data collected at the $750 \mathrm{~m}$ array were also derived using the $\mathrm{E}-\mathrm{W}$ method.
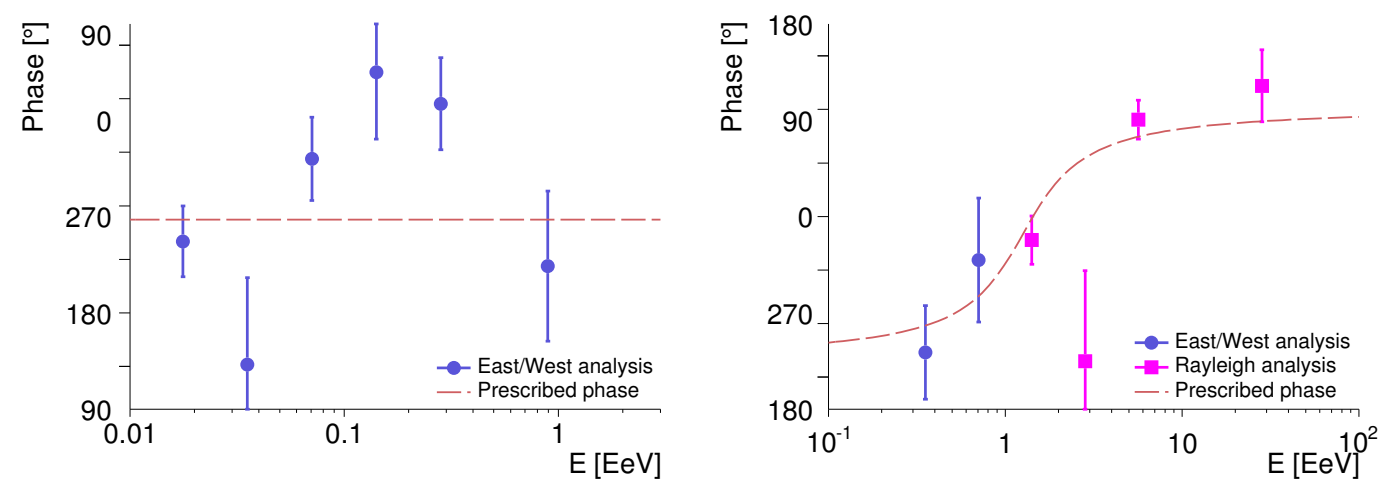

Figure 1: Left: Status of the prescription on phases for the $750 \mathrm{~m}$ array data using the E-W method, the prescribed test on phases $\left(\Phi=263^{\circ}\right)$ is shown with a dashed line. Right: Status of the prescription on phases for the $1500 \mathrm{~m}$ array data, the prescribed test on phases is shown with a dashed line.

Applying these particular analysis methods to data prior to 25 June 2011, a constant phase was observed around $\approx 270^{\circ}$ for energies below $1 \mathrm{EeV}$, while a change of phase to $\approx 100^{\circ}$ was observed at higher energies. This may be indicative of a real anisotropy, since a constancy of phases in ordered energy bins is expected to appear with a smaller number of events than necessary for the amplitudes to stand significantly above the background noise [9, 10]. Since the phases were not defined a priori, a prescribed test was set to establish with data posterior to 25 June 2011 whether this effect is real at $99 \% \mathrm{CL}$. Once an additional exposure of $21,000 \mathrm{~km}^{2} \mathrm{sr} \mathrm{yr}$ is reached for the $1500 \mathrm{~m}$ array, a positive anisotropy signal will be claimed with a global threshold of $1 \%$ upon the realization of one or two predefined conditions. The conditions require an alignment of phases 
detected by a likelihood ratio test with a chance probability less than $0.5 \%$ around a predefined phase value, assuming a signal amplitude of $0.5 \%$ for the $750 \mathrm{~m}$ array data and an amplitude comparable to the mean noise for the $1500 \mathrm{~m}$ array data over the whole energy range. From the date when the prescription started up to the end of 2014, the $750 \mathrm{~m}$ array has reached an exposure of $124 \mathrm{~km}^{2} \mathrm{sr} \mathrm{yr}$, while the $1500 \mathrm{~m}$ array has accumulated an exposure of $19,100 \mathrm{~km}^{2} \mathrm{sr} \mathrm{yr}$. The phases measured in each energy bin are shown in figure 1 for the $750 \mathrm{~m}$ array (left panel) and for the $1500 \mathrm{~m}$ array (right panel). At almost the end of the prescribed test, the phases do not appear aligned around the predefined values. The current p-values obtained from the likelihood test that the hypothesis of isotropy can account for the observations with no need of the alternative hypothesis are $40 \%$ for the $750 \mathrm{~m}$ array and $7 \%$ for the $1500 \mathrm{~m}$ array. Below $1 \mathrm{EeV}$, the phase alignment observed with data prior to June 2011 was suggestive of an overall signal amplitude at the percent level or so around $10^{17} \mathrm{eV}$. For lower amplitudes, such as $0.5 \%$ for instance, the current sensitivity of the analysis method to pick up a genuine alignment with a threshold of $0.5 \%$ is only $\simeq 50 \%$. Many more events and an improved sensitivity in the analysis method are thus required to reveal the large-scale structure of arrival directions in this energy range. On the other hand, the test making use of the $1500 \mathrm{~m}$ array data is challenged by the observed phase between $2 \times 10^{18} \mathrm{eV}$ and $4 \times 10^{18} \mathrm{eV}$ which value stands significantly far from the prescribed phase. Since the corresponding amplitude $\left(2.5 \times 10^{-3}\right)$ is lower than the mean noise $\left(6.7 \times 10^{-3}\right)$ expected from statistical fluctuations, the measurement of the phase in this energy bin is quite uncertain.

The final status of the prescribed test will be given once an additional exposure of 1,900 $\mathrm{km}^{2} \mathrm{sr} \mathrm{yr}$ is reached. This is expected from data recorded up to mid-2015.

\section{Dipole search above $4 \mathrm{EeV}$}

The Pierre Auger Collaboration have reported studies of the flux modulation in RA [2,9] as well as in both declination and right ascension [3,4] from the analysis of events with zenith angles smaller than $60^{\circ}$. Another study recently reported in [5] has been performed by including for the first time inclined events with zenith angles between $60^{\circ}$ and $80^{\circ}$, enabling the extension of the covered sky from $71 \%$ to $85 \%$. Large-scale angular modulations of the flux are studied by performing two Rayleigh analyses, one on the right ascension in the same manner as presented in section 2, and the other on the azimuth distribution, by replacing the expression of the Fourier coefficients given as a function of $\alpha$ in eqn. 2.2 by the azimuth angles $\varphi$, the latter being sensitive to modulations in declination.

The analysis has been performed in the two same energy bins as previous reports in this energy range. In the lower energy bin, between 4 and $8 \mathrm{EeV}$, harmonic coefficients are consistent with zero within their uncertainties, and there is no evidence for departures from isotropy in the right ascension distribution. In the highest energy bin where all events with energies greater than 8 $\mathrm{EeV}$ are gathered, the first harmonic has an amplitude $r_{1}=(4.4 \pm 1.0) \times 10^{-2}$, that has a chance probability $P\left(\geq r_{1}\right)=6.4 \times 10^{-5}$. The phase $\phi_{1}$ points to $95^{\circ} \pm 13^{\circ}$. The amplitude of the second harmonic is less significant, with a $2 \%$ probability of arising by chance. The azimuthal distribution after having accounted for the modulation induced by the tilt of the array and the geomagnetic effect - is expected to be uniform for energies above full efficiency for an isotropic distribution of CRs. The Fourier coefficients retrieved from the distribution in azimuth thus give information on the 
dipolar component along the Earth's rotation axis, $d_{z}$. The largest departure from isotropy appears for the $b_{1}^{\phi}$ Fourier coefficient in both energy bins. The negative values found indicate a dipolar component $d_{z}$ pointing to the south, although with low statistical significance $(4<E<8 \mathrm{EeV}$ : $2.4 \%, E>8 \mathrm{EeV}: 1.5 \%$ probability).

The reconstruction of the dipole components from the Rayleigh analysis has been done for the case where only a dipole contribution to large-scale anisotropies is relevant, and for the case where a possible quadrupole contribution is present. In the first case, the equatorial component $d_{\perp}$ is retrieved in the same manner as given in section 2, while the dipole component along the Earth rotation axis is retrieved through $d_{z}=b_{1}^{\phi} /\left(\cos \ell_{\mathrm{obs}}\langle\sin \theta\rangle\right)$, where $\ell_{\mathrm{obs}}$ denotes the latitude of the Observatory. The total dipole amplitude for the higher energy bin is $0.073 \pm 0.015$ pointing to $(\alpha, \delta)=\left(95^{\circ} \pm 13^{\circ},-39^{\circ} \pm 13^{\circ}\right)$. In the second case, a combination of a dipole plus a quadrupole was considered. It was found that the dipole is consistent with results from the first case with larger uncertainties, and the quadrupole components are not significant. The exposure-weighted average of the differential flux smoothed in angular windows of $45^{\circ}$ radius in equatorial coordinates is shown in figure $2{ }^{1}$ for the two energy bins considered. The maximum flux difference in the lower energy bin is just $8 \%$, while for the highest energy bin, it reaches $21 \%$.

$4 \mathrm{EeV}<\mathrm{E}<8 \mathrm{EeV}$

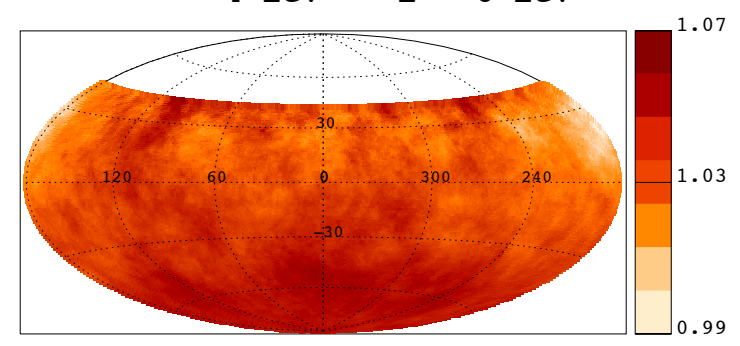

$\mathrm{E}>8 \mathrm{EeV}$

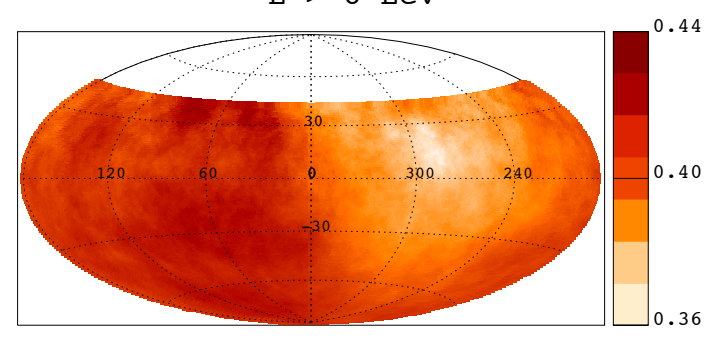

Figure 2: Sky map in equatorial coordinates of flux, in $\mathrm{km}^{-2} \mathrm{yr}^{-1} \mathrm{sr}^{-1}$ units, smoothed in angular windows of $45^{\circ}$ radius, for observed events with energies $4<E<8 \mathrm{EeV}$ (left) and $E>8 \mathrm{EeV}$ (right).

\section{Conclusion}

Different approaches have been explored by the Pierre Auger Collaboration to reveal largescale anisotropies imprinted on the CR arrival directions. These analyses take advantage of the large number of events provided by the two arrays, even below full detection efficiency. Using the cumulative data sets, a summary of these analyses is given in table 1 and figure 3 .

Upper limits on amplitudes are reported in the right panel of figure 3. In the two energy intervals where the p-values for the amplitudes are $1.5 \times 10^{-4}$ and $6.4 \times 10^{-5}$ (between 1 and $2 \mathrm{EeV}$, and for the integral bin above $8 \mathrm{EeV}$ (mean energy of $14.5 \mathrm{EeV}$ ) respectively, amplitudes are also shown. The observed amplitude above $8 \mathrm{EeV}$ suggests that a large-scale anisotropy is imprinted on the $\mathrm{CR}$ arrival directions of extragalactic $\mathrm{CRs}$ towards $\simeq 95^{\circ}$ in right ascension. It

\footnotetext{
${ }^{1} \mathrm{~A}$ rectification of the analogous figure published in [5] is shown here. Figure 3 in [5] had the flux incorrectly normalised to the exposure limited to events with zenith angles smaller than $60^{\circ}\left(37,142 \mathrm{~km}^{2} \mathrm{sr} \mathrm{yr}\right)$ while here it is correctly normalised to the total exposure including events with zenith angles between $60^{\circ}$ and $80^{\circ}\left(48,029 \mathrm{~km}^{2} \mathrm{sr} \mathrm{yr}\right)$.
} 

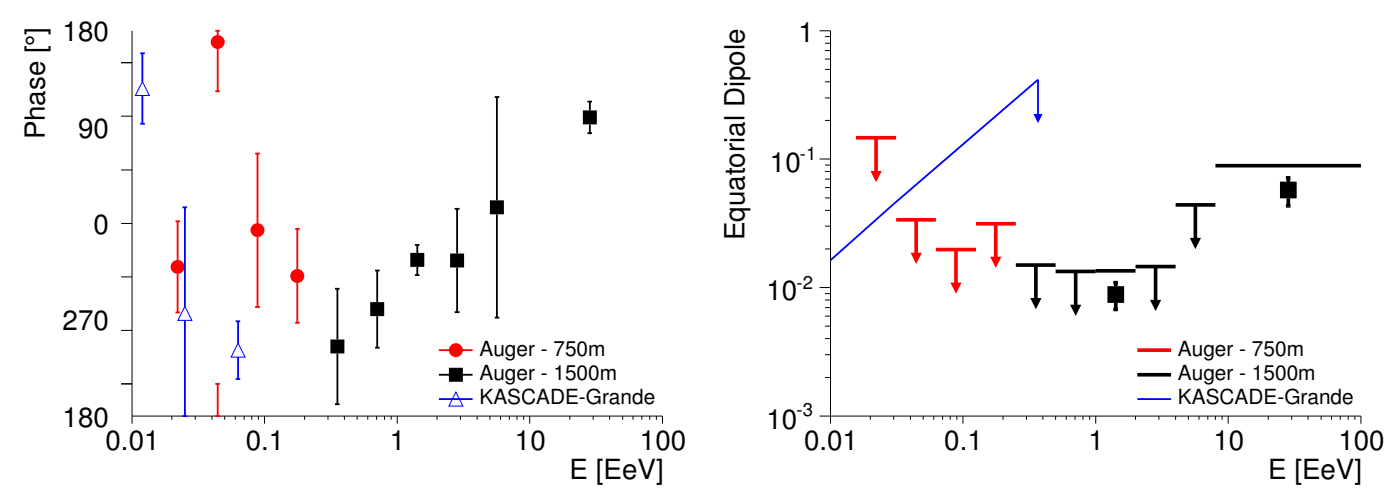

Figure 3: Left: Measured phases of the first harmonic modulation in RA. Right: Upper limits of the dipole equatorial component. Amplitudes are also reported in the two energy bins when the corresponding p-value expected from isotropy is below $10^{-3}$.

\begin{tabular}{c||c|c|c|c|c|c} 
& $\Delta E[\mathrm{EeV}]$ & $N$ & $d_{\perp} \pm \Delta_{d_{\perp}}[\%]$ & $\left.\phi \pm \Delta_{\phi}{ }^{\circ}\right]$ & $P\left(>d_{\perp}\right)[\%]$ & $d_{\perp}^{\mathrm{UL}}[\%]$ \\
\hline \hline $750 \mathrm{~m}[\mathrm{E}-\mathrm{W}]$ & $0.015-0.03$ & 32,244 & $6.4 \pm 3.8$ & $319 \pm 42$ & 25 & 14.5 \\
$750 \mathrm{~m}[\mathrm{E}-\mathrm{W}]$ & $0.03-0.06$ & 393,846 & $1.4 \pm 0.9$ & $169 \pm 46$ & 30 & 3.3 \\
$750 \mathrm{~m}[\mathrm{E}-\mathrm{W}]$ & $0.06-0.12$ & 581,313 & $0.5 \pm 0.6$ & $353 \pm 71$ & 73 & 2.0 \\
$750 \mathrm{~m}[\mathrm{E}-\mathrm{W}]$ & $0.12-0.25$ & 268,728 & $1.4 \pm 0.8$ & $310 \pm 43$ & 27 & 3.1 \\
$750 \mathrm{~m}[\mathrm{E}-\mathrm{W}]$ & $0.25-0.5$ & 68,782 & $2.8 \pm 1.5$ & $325 \pm 39$ & 20 & 6.0 \\
$750 \mathrm{~m}[\mathrm{E}-\mathrm{W}]$ & $0.5-1$ & 14,324 & $7.2 \pm 3.3$ & $233 \pm 31$ & 10 & 14.5 \\
\hline \hline $1500 \mathrm{~m}[\mathrm{E}-\mathrm{W}]$ & $0.25-0.5$ & 918,247 & $0.58 \pm 0.45$ & $245 \pm 54$ & 45 & 1.5 \\
$1500 \mathrm{~m}[\mathrm{E}-\mathrm{W}]$ & $0.5-1$ & $1,464,390$ & $0.65 \pm 0.33$ & $279 \pm 36$ & 15 & 1.3 \\
$1500 \mathrm{~m}[\mathrm{R}]$ & $1-2$ & 738,683 & $0.90 \pm 0.2$ & $326 \pm 14$ & $1.5 \times 10^{-2}$ & - \\
$1500 \mathrm{~m}[\mathrm{R}]$ & $2-4$ & 196,992 & $0.60 \pm 0.38$ & $325 \pm 48$ & 45 & 1.45 \\
$1500 \mathrm{~m} \mathrm{[R,*]}$ & $4-8$ & 50,417 & $0.40 \pm 0.80$ & $15 \pm 103$ & 88 & 4.0 \\
$1500 \mathrm{~m} \mathrm{[R,*]}$ & $>8$ & 19,797 & $5.7 \pm 1.3$ & $95 \pm 13$ & $6.4 \times 10^{-3}$ & -
\end{tabular}

Table 1: Summary of the harmonic analysis in different energy intervals in terms of the equatorial component of the dipole. In the left column, $[\mathrm{E}-\mathrm{W}]$ and $[\mathrm{R}]$ stand for the selected methods used to obtain the results, East-West or Rayleigh respectively. Data used are from 01/01/04 to 31/12/14, except for the two last bins indicated with $\left[R,{ }^{*}\right]$, where events with zenith angles larger than $60^{\circ}$ are included and where the ending date is $31 / 12 / 13$, as reported in [5].

is interesting to note that this phase is roughly in the opposite direction to the one suggested in the summary phase plot shown in the left panel for CRs with energies below $1 \mathrm{EeV}$ and which is in the general direction of the Galactic Centre. An interesting possibility to explain the low amplitudes over the wide energy range would thus be that a progressive cross-over might be taking place between a component of Galactic origin and another one of extragalactic origin. The global dipole anisotropy is then the sum of two vectors with opposite directions, providing then a natural mechanism to reduce significantly the amplitude of the vector describing the arrival directions of the whole population of CRs. Continued scrutiny of the large-scale distribution of arrival directions of CRs with increased sensitivity will provide further insights to reveal the origin of CRs in this 
energy range.

The measured dipolar anisotropy at the few $\%$ level above $8 \mathrm{EeV}$ could result from the diffusive propagation of extragalactic cosmic rays in the extragalactic turbulent magnetic field. This could happen if the amplitude of the field is large and/or if the cosmic rays have a component with large electric charge [13]. A large-scale anisotropy is also expected in the case that magnetic deflections are small if the cosmic ray sources are distributed similarly to the matter in the universe, due to the fact that in our local neighbourhood, matter is distributed inhomogeneously.

\section{References}

[1] The Pierre Auger Collaboration, The Pierre Auger Cosmic Ray Observatory, accepted for publication in Nucl. Instrum. Meth. A (2015) [arXiv:1502.01323].

[2] I. Sidelnik, for the Pierre Auger Collaboration, Measurement of the first harmonic modulation in the right ascension distribution of cosmic rays detected at the Pierre Auger Observatory: towards the detection of dipolar anisotropies over a wide energy range, Proc. 33rd ICRC, Rio de Janeiro, Brazil (2013) [arXiv:1307.5059].

[3] The Pierre Auger Collaboration, Large-scale Distribution of Arrival Directions of Cosmic Rays Detected Above $10^{18} \mathrm{eV}$ at the Pierre Auger Observatory, ApJS 203 (2012) 34 [arXiv:1210.3736].

[4] The Pierre Auger Collaboration, Constraints on the Origin of Cosmic Rays above $10^{18} \mathrm{eV} \mathrm{from}$ Large-scale Anisotropy Searches in Data of the Pierre Auger Observatory, ApJL 762 (2013) 13 [arXiv:1212.3083].

[5] The Pierre Auger Collaboration, Large scale distribution of ultra high energy cosmic rays detected at the Pierre Auger Observatory with zenith angles up to 80 $0^{\circ}$ ApJ 802 (2015) 111 [arXiv:1411.6953].

[6] J. Linsley, Fluctuation effects on directional data, Phys. Rev. Lett. 34 (1975) 1530.

[7] The Pierre Auger Collaboration, Atmospheric effects on extensive air showers observed with the surface detector of the Pierre Auger Observatory, Astropart. Phys. 32 (2009) 89 [arXiv:0906.5497].

[8] R. Bonino et al., The East-West Method: An Exposure-independent Method to Search for Large-scale Anisotropies of Cosmic Rays, ApJ 738 (2011) 67 [arXiv:1106.2651].

[9] The Pierre Auger Collaboration, Search for first harmonic modulation in the right ascension distribution of cosmic rays detected at the Pierre Auger Observatory, Astropart. Phys. 34 (2011) 627 [arXiv:1103.2721]

[10] D. Edge et al., A study of the arrival direction distribution of high-energy particles as observed from the Northern Hemisphere, J. Phys. G 4 (1978) 133.

[11] F. J. M. Farley \& J. R. Storey, The Sidereal Correlation of Extensive Air Showers, Proc. Phys. Soc. A 67 (1954) 996.

[12] The Pierre Auger Collaboration, Reconstruction of inclined air showers detected with the Pierre Auger Observatory, JCAP 08 (2014) 019 [arXiv:1407.3214]

[13] D. Harari, S. Mollerach, E. Roulet, Anisotropies of ultrahigh energy cosmic rays diffusing from extragalactic sources, Phys. Rev. D 89 (2014) 123001 [arXiv:1312.1366] 